\title{
First Order Partial Differential Equations \\ Containing a Small Parameter*
}

\author{
J. J. LEVIN
}

Purdue University, Lafayette, Indiana

Communicated by D. GilbarG

1. Introduction. The equation investigated here is

$$
\epsilon\left\{a_{1}(x, y, u, \epsilon) \frac{\partial u}{\partial x}+a_{2}(x, y, u, \epsilon) \frac{\partial u}{\partial y}\right\}=b(x, y, u, \epsilon)
$$

where $\epsilon$ is a small positive parameter and all the functions and variables appearing are real scalars. Conditions will be given under which the solutions of (1.1) passing through a fixed initial curve, $\Gamma$, where

$$
\Gamma:\left\{\begin{array}{l}
x=f_{1}(\tau) \\
y=f_{2}(\tau), \\
u=f_{3}(\tau)
\end{array} \quad \tau_{0} \leqq \tau \leqq \tau_{1} ;\right.
$$

approach the surface $b(x, y, u, 0)=0$ as $\epsilon \rightarrow 0+$.

Our main result is Theorem 1, which is expressed in terms of (1.1) and is given below. In order to prove Theorem 1 it is convenient to transform (1.1) to a somewhat simpler form, (2.10). Theorem 1 is then restated, as Theorem 2 , in terms of (2.10); this is done in Section 2. Some lemmas that are used repeatedly in the proofs are given in Section 3. In Section 4 the characteristic equations associated with (2.10) are investigated, and in Section 5, Theorem 2 is proven. A boundary layer equation associated with (2.10) is considered in Section 6 .

The analysis centers around the characteristic curves associated with (2.10)

* This paper was written in part in the course of work sponsored by the Office of Naval Research. The author is indebted to Professor Norman Levinson for many helpful suggestions. 
and is similar to that employed in [2] on an analogous problem in ordinary differential equations.

The following assumptions concerning (1.1) and (1.2) are made:

$\mathrm{H1}$ : Hypotheses on $b(x, y, u, \epsilon)$ ((iv) is the crucial one).

(i) There exists a function $h(x, y)$ defined in some non-vacuous simply connected closed region, $Q$, of the $x, y$-plane, such that $b(x, y, h(x, y), 0) \equiv 0$ for $(x, y) \varepsilon Q$. It is further assumed that the boundary of $Q$ has a continuously turning tangent.

(ii) $h$ is of class $C^{(3)}$ in $Q$.

(iii) There exist constants $\delta_{0}>0$ and $\epsilon_{0}>0$ such that in the region $R$ of the $x, y, u, \epsilon$ space defined by

$$
\mathrm{R}: h(x, y)-\delta_{0}<u<h(x, y)+\delta_{0}, \quad(x, y) \varepsilon Q ; \quad 0 \leqq \epsilon<\epsilon_{0}
$$

$b$ and its second partial derivatives with respect to $x, y, u$ are continuous in $(x, y, u, \epsilon)$.

(iv) There exists a constant $\mu>0$ such that

$$
\text { In } Q: b_{u}(x, y, h(x, y), 0) \leqq-\mu .
$$

H2: Hypotheses on $a_{i}(x, y, u, \epsilon), i=1,2$.

(i) In $R: a_{i}$ and their third partial derivatives with respect to $x, y, u$ are continuous in $(x, y, u, \epsilon), \mathrm{i}=1,2$.

(ii) In $Q: \bar{a}_{1}^{2}(x, y)+\bar{a}_{2}^{2}(x, y)>0$, where $\bar{a}_{i}(x, y)=a_{i}(x, y, h(x, y), 0), i=1,2$. H3: Hypotheses on $\Gamma$. (The projection of $\Gamma$ on the $x, y$-plane is denoted by $\Gamma^{\prime}$.)

(i) $f_{i}^{(3)}(\tau) \varepsilon C\left(\tau_{0} \leqq \tau \leqq \tau_{1}\right), \quad i=1,2 ; \quad f_{3}^{\prime}(\tau) \varepsilon C\left(\tau_{0} \leqq \tau \leqq \tau_{1}\right)$.

(ii) $\Gamma^{\prime}$ lies in the interior of $Q$ and has no double points.

(iii) $\left(f_{1}(\tau), f_{2}(\tau), f_{3}(\tau), 0\right)$ lies in the interior of $R$ for $\tau_{0} \leqq \tau \leqq \tau_{1}$.

(iv) $a_{1}\left(f_{1}(\tau), f_{2}(\tau), q(\tau), 0\right) f_{2}^{\prime}(\tau)-a_{2}\left(f_{1}(\tau), f_{2}(\tau), q(\tau), 0\right) f_{1}^{\prime}(\tau) \neq 0$,

where $q(\tau)=h\left(f_{1}(\tau), f_{2}(\tau)\right)$.

$$
\tau_{0} \leqq \tau \leqq \tau_{1}
$$

(v) None of the solution curves of the system

$$
\frac{d x}{d \sigma}=\bar{a}_{1}(x, y), \quad \frac{d y}{d \sigma}=\bar{a}_{2}(x, y)
$$

which pass through $\Gamma^{\prime}$ approach the boundary of $Q$ tangentially.

Theorem 1. H1, H2, and $\mathrm{H} 3$ imply that there exist a non-vacuous closed region, $D$ (which is independent of $\epsilon$ ), in the $x, y$-plane and constants $\hat{\delta}>0$ and $\hat{\epsilon}>0$ (both independent of $\epsilon$ ), such that if $\left|q(\tau)-f_{3}(\tau)\right| \leqq \hat{\delta}$ for $\tau_{0} \leqq \tau \leqq \tau_{1}$, then (1.1) has a unique solution $u=H(x, y, \epsilon)$ which is defined over $D$ and satisfies the initial condition $H\left(f_{1}(\tau), f_{2}(\tau), \epsilon\right)=f_{3}(\tau)$ for $\tau_{0} \leqq \tau \leqq \tau_{1}, 0<\epsilon \leqq \hat{\epsilon}$. Furthermore, $H(x, y, \epsilon) \rightarrow h(x, y), \partial H(x, y, \epsilon) / \partial x \rightarrow \partial h(x, y) / \partial x$, and $\partial H(x, y, \epsilon) / \partial y \rightarrow$ $\partial h(x, y) / \partial y$ as $\epsilon \rightarrow 0+$ uniformly over every subset of $D$ which is a positive distance from $\Gamma^{\prime}$.

Remark on the region $D$ of Theorem 1 . It is clear, from the characteristic equa- 
tions associated with (1.1), that in general the domain of definition of the solution of (1.1) depends on $\epsilon$. However, the dependence of this domain, denoted by $D(\epsilon)$, on $\epsilon$ is such that

$$
\bigcap_{\epsilon>0}^{e=\epsilon} D(\epsilon) \supset D .
$$

We have taken some of our hypotheses for the purpose of obtaining, in a certain sense, a maximum size for $D$. A more local result could be obtained under more general hypotheses. The precise definition of $D$ will be found in a remark following the statement of Theorem 2.

2. Transformation of (1.1). For the purpose of transforming (1.1) to a simpler form, we shall use the system

$$
\frac{d x}{d \sigma}=\bar{a}_{1}(x, y), \quad \frac{d y}{d \sigma}=\bar{a}_{2}(x, y)
$$

of H3(v) to set up a curvilinear coordinate system in a certain subset of $Q$ which will be denoted by $Q_{1}$. $Q_{1}$ will be taken sufficiently large so as to include all the $D(\epsilon)$ (and hence also $D$ ) for $\epsilon$ sufficiently small. Let

$$
x=\phi(\sigma, \tau), \quad y=\psi(\sigma, \tau)
$$

denote the solution of (2.1) which satisfies the initial condition

$$
\phi(0, \tau)=f_{1}(\tau), \quad \psi(0, \tau)=f_{2}(\tau), \quad \tau_{0}-\lambda \leqq \tau \leqq \tau_{1}+\lambda,
$$

where $\lambda>0$. In (2.3) we have assumed that $\Gamma^{\prime}$ has been extended in both directions so that the new curve satisfies $\mathrm{H} 3$ in the larger interval $\tau_{0}-\lambda \leqq \tau \leqq \tau_{1}+\lambda$. Simple continuity considerations show that this is possible for some $\lambda>0$. The continuity in $(\sigma, \tau)$ of the third partial derivatives of $\phi(\sigma, \tau)$ and $\psi(\sigma, \tau)$ is clear from well known theorems.

In order to define $Q_{1}$ we first define a function $Z(\tau)$ and a closed region $S$ of the $\sigma, \tau$-plane as follows:

$$
Z(\tau)=\lim _{P \rightarrow B} \sigma(P), \quad \tau_{0}-\lambda \leqq \tau \leqq \tau_{1}+\lambda,
$$

where $P$ is the point $(\phi(\sigma, \tau), \psi(\sigma, \tau))$ and $B=B(\tau)$ is the point on the boundary of $Q$ that $P$ approaches as $\sigma$ increases positively. Note that $Z(\tau) \varepsilon C^{\prime}$ $\left(\tau_{0}-\lambda \leqq \tau \leqq \tau_{1}+\lambda\right)$.

$$
S: \quad 0 \leqq \sigma \leqq Z(\tau), \quad \tau_{0}-\lambda \leqq \tau \leqq \tau_{1}+\lambda .
$$

Finally, we define $Q_{1}$ as the image of $S$ in the $x, y$-plane under the mapping (2.2). Thus $Q_{1}$ is a curvilinear quadrilateral whose sides are the extended $\Gamma^{\prime}$, the curves $\tau=\tau_{0}-\lambda$ and $\tau=\tau_{1}+\lambda$ of (2.2), and the arc of the boundary of $Q$ intercepted by these two curves of (2.2). 
The proof that (2.2) defines a curvilinear coordinate system $\sigma, \tau$ in $Q_{1}$ shall now be completed by showing that the Jacobian $\Delta(\sigma, \tau) \neq 0$ in $S$, where

$$
\Delta(\sigma, \tau)=\frac{\partial \phi}{\partial \sigma} \frac{\partial \psi}{\partial \tau}-\frac{\partial \phi}{\partial \tau} \frac{\partial \psi}{\partial \sigma}
$$

A straightforward computation shows that

$$
\frac{\partial \Delta}{\partial \sigma}=\left(\frac{\partial \bar{a}_{1}}{\partial x}+\frac{\partial \bar{a}_{2}}{\partial y}\right) \Delta .
$$

From H3(iv) we have $\Delta(0, \tau) \neq 0$, which together with (2.5) immediately yields the non-vanishing of $\Delta(\sigma, \tau)$.

Equation (1.1) is now written in terms of the $\sigma, \tau$ coordinates. The elementary relations

$$
u_{x}=\frac{1}{\Delta}\left\{u_{\sigma} \psi_{\tau}-u_{\tau} \psi_{\sigma}\right\}, \quad u_{y}=\frac{1}{\Delta}\left\{u_{\tau} \phi_{\sigma}-u_{\sigma} \phi_{\tau}\right\}
$$

and the definition

$$
v=u-h
$$

are used to obtain

$$
\epsilon\left\{\tilde{a}_{1}(\sigma, \tau, v, \epsilon) \frac{\partial v}{\partial \sigma}+\tilde{a}_{2}(\sigma, \tau, v, \epsilon) \frac{\partial v}{\partial \tau}\right\}=\tilde{b}(\sigma, \tau, v, \epsilon)
$$

where

$$
\begin{aligned}
& \tilde{a}_{1}=\frac{1}{\Delta}\left\{a_{1}(\phi, \psi, v+h, \epsilon) \psi_{\tau}-a_{2}(\phi, \psi, v+h, \epsilon) \phi_{\tau}\right\} \\
& \tilde{a}_{2}=\frac{1}{\Delta}\left\{-a_{1}(\phi, \psi, v+h, \epsilon) \psi_{\sigma}+a_{2}(\phi, \psi, v+h, \epsilon) \phi_{\sigma}\right\} \\
& \tilde{b}=b(\phi, \psi, v+h, \epsilon)-\epsilon\left\{\tilde{a}_{1} h_{\sigma}+\tilde{a}_{2} h_{\tau}\right\} .
\end{aligned}
$$

Equation (2.6) is meaningful in the set $\tilde{R}$ of $\sigma, \tau, v, \epsilon$-space, where

$$
\tilde{R}: \quad(\sigma, \tau) \varepsilon S,|v|<\delta_{0} ; \quad 0 \leqq \epsilon<\epsilon_{0} .
$$

The initial curve for (2.6) is given by

$$
\tilde{\Gamma}:\left\{\begin{array}{l}
\sigma=0 \\
\tau=t, \\
v=f_{3}(t)-q(t)
\end{array} \quad t_{0} \leqq t \leqq t_{1} .\right.
$$


From $\mathrm{H}_{2},(2.1)$, and (2.4) one has

$$
\tilde{a}_{1}(\sigma, \tau, 0,0)=1, \quad \tilde{a}_{2}(\sigma, \tau, 0,0)=0 .
$$

Therefore

$$
\epsilon\left\{\frac{\partial v}{\partial \sigma}+a^{*}(\sigma, \tau, v, \epsilon) \frac{\partial v}{\partial \tau}\right\}=b^{*}(\sigma, \tau, v, \epsilon)
$$

where

$$
a^{*}=\tilde{a}_{2} / \tilde{a}_{1}, \quad b^{*}=\tilde{b} / \tilde{a}_{1},
$$

is equivalent to (2.6) in $\tilde{R}$ if $\delta_{0}$ and $\epsilon_{0}$ are sufficiently small. It is easily verified that

$$
\begin{aligned}
& a^{*}(\sigma, \tau, 0,0)=0 \\
& b^{*}(\sigma, \tau, 0,0)=0 \\
& b_{v}^{*}(\sigma, \tau, 0,0) \leqq-\mu .
\end{aligned}
$$

$\tilde{\Gamma}$ is also the initial curve for (2.8).

Thus we have transformed (1.1) with an initial curve (1.2) to an equation of the form

$$
\epsilon\left\{\frac{\partial u}{\partial x}+a(x, y, u, \epsilon) \frac{\partial u}{\partial y}\right\}=b(x, y, u, \epsilon)
$$

with an initial curve of the form

$$
\Gamma:\left\{\begin{array}{l}
x=0 \\
y=t, \\
u=\gamma(t)
\end{array} \quad t_{0} \leqq t \leqq t_{1} .\right.
$$

Furthermore, in certain regions $Q$ and $R$ of the $x, y$-plane and $x, y, u, \epsilon$-space respectively, defined by

$$
\begin{aligned}
& Q: \quad 0 \leqq x \leqq Z(y), \quad t_{0}-\lambda \leqq y \leqq t_{1}+\lambda \\
& R: \quad(x, y) \varepsilon Q,|u|<\delta_{0} ; \quad 0 \leqq \epsilon<\epsilon_{0},
\end{aligned}
$$

where $\lambda, \delta_{0}$, and $\epsilon_{0}$ are positive constants and $Z(y)$ is a continuously differentiable positive function, $a(x, y, u, \epsilon), b(x, y, u, \epsilon)$, and $\Gamma$ satisfy the following hypotheses:

$\mathrm{H} 1^{\prime}:$ (i) In $Q: b(x, y, 0,0) \equiv 0$.

(ii) In $R: b(x, y, u, \epsilon)$ and its second partial derivatives with respect to $x, y, u$ are continuous in $(x, y, u, \epsilon)$.

(iii) In $Q: b_{u}(x, y, 0,0) \leqq-\mu<0$. 
$\mathrm{H}^{\prime}$ : (i) In $R: a(x, y, u, \epsilon)$ and its second partial derivatives with respect to $x, y, u$ are continuous in $(x, y, u, \epsilon)$.

(ii) In $Q: a(x, y, 0,0) \equiv 0$.

$\mathrm{H}^{\prime}:$ (i) $\gamma^{\prime}(t) \varepsilon C\left(t_{0} \leqq t \leqq t_{1}\right)$.

(ii) $(0, t, \gamma(t), 0)$ lies in the interior of $R$ for $t_{0} \leqq t \leqq t_{1}$.

Theorem 2. $\mathrm{H}^{\prime}, \mathrm{H}^{\prime}$, and $\mathrm{H}^{\prime}$ imply that there exists a closed non-vacuous region, $D$ (which is independent of $\epsilon$ ), in the $x, y$-plane and constants $\hat{\delta}>0$ and $\hat{\boldsymbol{\epsilon}}>0$ (both independent of $\epsilon$ ), such that if $|\gamma(t)| \leqq \hat{\delta}$ for $t_{0} \leqq t \leqq t_{1}$, then (2.10) has a unique solution $u=H(x, y, \epsilon)$ which is defined over $D$ and satisfies the initial condition $H(0, t, \epsilon)=\gamma(t)$ for $t_{0} \leqq t \leqq t_{1}, 0<\epsilon \leqq \hat{\epsilon}$. Furthermore, $H(x, y, \epsilon) \rightarrow$ $0, \partial H(x, y, \epsilon) / \partial x \rightarrow 0$, and $\partial H(x, y, \epsilon) / \partial y \rightarrow 0$ as $\epsilon \rightarrow 0+$ uniformly for $x \geqq C$ for any $C>0$.

Definition of the region $D$ of Theorem 2 . Let $\zeta$ be a positive number sufficiently small that the lines

$$
l_{1}: y=t_{0}+\zeta x, \quad l_{2}: y=t_{1}-\zeta x
$$

do not intersect in $Q ; \zeta$ is then fixed. Then $D$ is defined to be the region bounded by the line segment $t_{0} \leqq y \leqq t_{1}$ on the $y$-axis, the lines $l_{1}$ and $l_{2}$, and the arc of the curve $x=Z(y)$ intercepted by $l_{1}$ and $l_{2}$.

Remarks. (i) The remark following Theorem 1 concerning (1.1) is now applicable to (2.10).

(ii) The lines $l_{1}$ and $l_{2}$ appearing in the definition of $D$ could be replaced by the lines $y=t_{0}$ and $y=t_{1}$ respectively. However, this would force us to extend the interval on which $\gamma(t)$ is defined and thereby lose the uniqueness of Theorem 2.

(iii) The $D$ of Theorem 1 is obtained from the $D$ of Theorem 2 with the aid of the transformation (2.2).

\section{Preliminary lemmas.}

Lemma 3.1 Let $\rho(s)$ and $\alpha(s)$ be real integrable functions of $s$ on $s_{0} \leqq s \leqq s_{1}$ and let $\lambda$ be any positive number. If

$$
\rho(s) \leqq \alpha(s)+\lambda \int_{s_{0}}^{s} \rho(\tau) d \tau, \quad s_{0} \leqq s \leqq s_{1},
$$

then

$$
\rho(s) \leqq \alpha(s)+\lambda \int_{s_{0}}^{s} e^{\lambda(s-\tau)} \alpha(\tau) d \tau, \quad \quad s_{0} \leqq s \leqq s_{1}
$$

Proof. See [2].

Lemma 3.2 Let $\rho(s)$ be a real function of $s$ possessing an integrable derivative on $s_{0} \leqq s \leqq s_{1}$. Let $\alpha(s)$ be a real integrable function of $s$ on $s_{0} \leqq s \leqq s_{1}$, and let $\lambda$ be any positive number. If

$$
\left|\frac{d \rho}{d s}\right| \leqq \alpha(s)+\lambda|\rho|, \quad s_{0} \leqq s \leqq s_{1},
$$


then

$$
|\rho(s)| \leqq\left|\rho\left(s_{0}\right)\right| e^{\lambda\left(s-s_{0}\right)}+\int_{s_{0}}^{s} e^{\lambda(s-\tau)} a(\tau) d \tau, \quad s_{0} \leqq s \leqq s_{1} .
$$

Proof. See [2].

Lemma 3.3. If $\alpha(s)$ and $\beta(s) \varepsilon C\left(s_{0} \leqq s \leqq s_{1}\right)$, then the solution of

$$
\frac{d w}{d s}=\alpha(s) w+\beta(s), \quad s_{0} \leqq s \leqq s_{1},
$$

which satisfies the initial condition

$$
w\left(s_{0}\right)=w_{0},
$$

is

$(3.2) w(s)=w_{0} \exp \left(\int_{s_{0}}^{s} \alpha(\tau) d \tau\right)+\int_{s_{0}}^{s} \exp \left(\int_{\xi}^{s} \alpha(\tau) d \tau\right) \beta(\xi) d \xi, \quad s_{0} \leqq s \leqq s_{1}$.

Proof. Substitute (3.2) into (3.1).

4. The characteristics. The characteristic equations associated with (2.10) are

$$
\begin{aligned}
\frac{d x}{d s} & =1 \\
\frac{d y}{d s} & =a(x, y, u, \epsilon) \\
\epsilon \frac{d u}{d s} & =b(x, y, u, \epsilon) .
\end{aligned}
$$

The existence, uniqueness, and behavior as $\epsilon \rightarrow 0+$ of the solutions of (4.1) and their first partial derivatives with respect to $s$ and $t$ will be investigated in this section.

Lemma 4.1. There exist constants $\delta_{1}$ and $\epsilon_{1}$, which are independent of $\epsilon$ and satisfy the inequalities

$$
0<\delta_{1}<\delta_{0}, \quad 0<\epsilon_{1}<\epsilon_{0},
$$

such that if

$$
|\gamma(t)| \leqq \delta_{1}, \quad t_{0} \leqq t \leqq t_{1},
$$

then (4.1) has a unique solution

$$
\begin{aligned}
& x=r(s, t, \epsilon) \equiv s \\
& y=p(s, t, \epsilon) \\
& u=g(s, t, \epsilon)
\end{aligned}
$$


satisfying the initial conditions

$$
\begin{aligned}
& r(0, t, \epsilon)=0 \\
& p(0, t, \epsilon)=t, \quad t_{0} \leqq t \leqq t_{1} . \\
& g(0, t, \epsilon)=\gamma(t)
\end{aligned}
$$

Furthermore, $p$ and $g$ exist and satisfy the inequalities

$$
\begin{aligned}
& |p(s, t, \epsilon)-t| \leqq \omega(\epsilon) \\
& |g(s, t, \epsilon)| \leqq 2|\gamma(t)| e^{-\mu s / 2 \epsilon}+\omega(\epsilon)
\end{aligned}
$$

for $t_{0} \leqq t \leqq t_{1}, 0<\epsilon \leqq \epsilon_{1}$, where

$$
\omega(\epsilon) \varepsilon C\left(0 \leqq \epsilon \leqq \epsilon_{1}\right), \omega(0)=0 .
$$

Proof. It is evident from classical existence and uniqueness theorems that for any fixed $t,(4.1)$ has a unique solution (4.2) which satisfies (4.3) and which exists on some $s$ interval (which may well depend on $\epsilon$ ) to the right of $s=0$. However, it follows from (4.4) that for sufficiently small $\epsilon_{1}$ the curves $x=s$, $y=p(s, t, \epsilon)$ may, for each fixed $t$, be extended until they reach the boundary curve $x=Z(y)$ of $Q$.

Whenever they appear $x, y$, and $u$ represent $r, p$, and $g$ respectively. Clearly

$$
x \equiv s .
$$

The third equation of (4.1) may be written as

$$
\epsilon \frac{d u}{d s}=B(s, t) u+G_{1}(s, t, \epsilon)+G_{2}(s, t)
$$

where

$$
\begin{aligned}
& B(s, t)=b_{u}(s, t, 0,0) \\
& G_{1}(s, t, \epsilon)=b(s, y, u, \epsilon)-b(s, t, u, 0) \\
& G_{2}(s, t)=b(s, t, u, 0)-b(s, t, 0,0)-b_{u}(s, t, 0,0) u .
\end{aligned}
$$

In (4.8) we have used $b(s, t, 0,0) \equiv 0$. Dividing (4.7) by $\epsilon$ and applying Lemma 3.3 to the resulting equation yields

$$
\begin{aligned}
u(s, t, \epsilon)=\gamma(t) \exp & \left(\frac{1}{\epsilon} \int^{0} B(\xi, t) d \xi\right) \\
+ & \frac{1}{\epsilon} \int_{0}^{s} \exp \left(\frac{1}{\epsilon} \int_{\xi}^{s} B(\eta, t) d \eta\right)\left\{G_{1}(\xi, t, \epsilon)+G_{2}(\xi, t)\right\} d \xi .
\end{aligned}
$$

Equation (4.9), of course, is valid as long as $(x, y, u, \epsilon)$ remains in $R$. 
For simplicity of notation we adopt the following two conventions: The letter $K$ shall always denote a finite positive real number which does not depend on $s, t$, or $\epsilon$. Furthermore, while $K$ may represent different constants in different formulae, $K$ shall be a well-determined number at each stage of the proof. The function $\omega=\omega(\epsilon)$ shall always denote a non-negative continuous function of $\epsilon$, defined for $\epsilon$ sufficiently small (exactly how small will always be stated), which does not depend on $s$ or $t$ and such that $\omega(0)=0$. Furthermore, while $\omega(\epsilon)$ may represent different functions in different formulae, $\omega(\epsilon)$ shall be a well-determined function at each stage of the proof. (Note that on the basis of these conventions $K \omega(\epsilon)=\omega(\epsilon)$.)

Let $\tilde{\delta}_{0}$ and $\tilde{\epsilon}_{0}$ satisfy $0<\tilde{\delta}_{0}<\delta_{0}$ and $0<\tilde{\epsilon}_{0}<\epsilon_{0}$ respectively; hold $\tilde{\delta}_{0}$ and $\tilde{\epsilon}_{0}$ fixed throughout the entire proof. Let $\nu>0$. From $\mathrm{H}^{\prime}{ }^{\prime}$ there exists a constant $\tilde{\delta}_{1}=\tilde{\delta}_{1}(\nu)$, satisfying $0<\tilde{\delta}_{1} \leqq \tilde{\delta}_{0}$ and such that

$$
\begin{aligned}
& \left|G_{1}(s, t, \epsilon)\right| \leqq K|y(s, t, \epsilon)-t|+\omega(\epsilon) \\
& \left|G_{2}(s, t)\right| \leqq \nu|u(s, t, \epsilon)| \\
& \text { if }(x, y) \varepsilon Q, \quad t_{0} \leqq t \leqq t_{1}, \quad|u| \leqq \tilde{\delta}_{1}, \quad 0<\epsilon \leqq \tilde{\epsilon}_{0} .
\end{aligned}
$$

From $\mathrm{H1}^{\prime}$, (4.9), and (4.10) one readily obtains

$$
\begin{gathered}
|u(s, t, \epsilon)| \leqq \\
+\gamma(t) \mid e^{-\mu s / \epsilon}+\omega(\epsilon) \\
+\frac{K}{\epsilon} \int_{0}^{s} e^{-\mu(s-\xi) / \epsilon}|y(\xi, t, \epsilon)-t| d \xi \\
+\frac{\nu}{\epsilon} \int_{0}^{s} e^{-\mu(s-\xi) / \epsilon}|u(\xi, t, \epsilon)| d \xi \\
\quad t_{0} \leqq t \leqq t_{1}, \quad|u| \leqq \tilde{\delta}_{1}, \quad 0<\epsilon \leqq \tilde{\epsilon}_{0} . \\
\text { With the aid of the second equation of }(4.1) \text { we shall now show that } \\
\quad I=\frac{1}{\epsilon} \int_{0}^{s} e^{-\mu(s-\xi) / \epsilon}|y(\xi, t, \epsilon)-t| d \xi \leqq \frac{1}{\mu}|y(s, t, \epsilon)-t|+K \epsilon \\
\text { if }(x, y) \varepsilon Q, \quad t_{0} \leqq t \leqq t_{1}, \quad|u| \leqq \tilde{\delta}_{0}, \quad 0<\epsilon \leqq \tilde{\epsilon}_{0} .
\end{gathered}
$$

Clearly there exists a $K>0$ such that

$$
\begin{gathered}
\left|\frac{\partial y}{\partial s}(s, t, \epsilon)\right| \leqq K \\
\text { if } \quad(x, y,) \varepsilon Q, \quad t_{0} \leqq t \leqq t_{1}, \quad|u| \leqq \tilde{\delta}_{0}, \quad 0<\epsilon \leqq \tilde{\boldsymbol{\epsilon}}_{0} ;
\end{gathered}
$$

hence, by the mean value theorem,

$$
\begin{aligned}
& |y(s, t, \epsilon)-y(\xi, t, \epsilon)| \leqq K|s-\xi| \\
& \text { if }(s, y) \text { and }(\xi, y) \varepsilon Q, \quad t_{0} \leqq t \leqq t_{1}, \quad|u| \leqq \tilde{\delta}_{0}, \quad 0<\epsilon \leqq \tilde{\epsilon}_{0} .
\end{aligned}
$$


But

$$
I \leqq I_{1}+I_{2}
$$

where

$$
\begin{aligned}
& I_{1}=\frac{1}{\epsilon}|y(s, t, \epsilon)-t| \int_{0}^{s} e^{-\mu(s-\xi) / \epsilon} d \xi \\
& I_{2}=\frac{1}{\epsilon} \int_{0}^{s} e^{-\mu(s-\xi) / \epsilon}|y(s, t, \epsilon)-y(\xi, t, \epsilon)| u \xi
\end{aligned}
$$

Now

$$
I_{1} \leqq \frac{1}{\mu}|y(s, t, \epsilon)-t|
$$

and by (4.13)

$$
I_{2} \leqq \frac{K}{\epsilon} \int_{0}^{s} e^{-\mu(s-\xi) / \epsilon}(s-\xi) d \xi
$$

The change of variables $\eta=\mu(s-\xi) / \epsilon$ yields

$$
I_{2} \leqq K \epsilon \text {. }
$$

Combining (4.14) and (4.15) we see that we have proven (4.12).

Substituting (4.12) into (4.11) yields

$$
|u(s, t, \epsilon)| \leqq|\gamma(t)| e^{-\mu s / \epsilon}+K|y(s, t, \epsilon)-t|+\omega(\epsilon)
$$

$$
+\frac{\nu}{\epsilon} \int_{0}^{s} e^{-\mu(s-\xi) / \epsilon}|u(\xi, t, \epsilon)| d \xi
$$

$$
\text { if }(x, y) \varepsilon Q, \quad t_{0} \leqq t \leqq t_{1}, \quad|u| \leqq \tilde{\delta}_{1}, \quad 0<\epsilon \leqq \tilde{\epsilon}_{0} .
$$

Multiplying both sides of (4.16) by $e^{\mu s / \epsilon}$, setting

$$
F(s, t, \epsilon)=e^{\mu s / \epsilon}|u(s, t, \epsilon)|
$$

in the resulting inequality, and then applying Lemma 3.1 results in

$$
\begin{aligned}
&|u(s, t, \epsilon)| \leqq|\gamma(t)| e^{-\mu s / \epsilon}+K|y(s, t, \epsilon)-t|+\omega(\epsilon) \\
&+\frac{\nu}{\epsilon} e^{-\mu s / \epsilon}|\gamma(t)| \int_{0}^{s} e^{\nu(s-\xi) / \epsilon} d \xi \\
&+\frac{\nu}{\epsilon} \int_{0}^{s} e^{-(\mu-\nu)(s-\xi) / \epsilon}\{K|y(\xi, t, \epsilon)-t|+\omega(\epsilon)\} d \xi \\
& \text { if }(x, y) \varepsilon Q, \quad t_{0} \leqq t \leqq t_{1}, \quad|u| \leqq \tilde{\delta}_{1}, \quad 0<\epsilon \leqq \tilde{\epsilon}_{0} .
\end{aligned}
$$


We now ehoose $\nu>0$ so that $\mu-\nu>\frac{1}{2} \mu$ and then hold $\nu$ fixed at this value, which we denote by $\nu_{0}$. This determines a $\tilde{\delta}_{1}$ which is then also held fixed. After some elementary integrations and application of an inequality similar to (4.12) we obtain

$$
\begin{aligned}
& |u(s, t, \epsilon)| \leqq 2|\gamma(t)| e^{-\mu s / 2 \epsilon}+K|y(s, t, \epsilon)-t|+\omega(\epsilon) \\
& \text { if }(x, y) \varepsilon Q, \quad t_{0} \leqq t \leqq t_{1}, \quad|u| \leqq \tilde{\delta}_{1}, \quad 0<\epsilon \leqq \tilde{\epsilon}_{0} .
\end{aligned}
$$

From the second equation of (4.1) and (4.6) one has

$$
\frac{d y}{d s}=a(s, y, u, \epsilon)
$$

from which

$$
\frac{d}{d s}(y-t)=a(s, y, u, \epsilon)
$$

and, hence, by $\mathrm{H}_{2}^{\prime}$

$$
\begin{gathered}
\left|\frac{d}{d s}(y-t)\right| \leqq K\{|y-t|+|u|\}+\omega(\epsilon) \\
\text { if }(x, y) \varepsilon Q, \quad t_{0} \leqq t \leqq t_{1}, \quad|u| \leqq \tilde{\delta}_{0}, \quad 0<\epsilon \leqq \tilde{\epsilon}_{0} .
\end{gathered}
$$

Substitution of (4.18) into (4.19) results in

$$
\begin{gathered}
\left|\frac{d}{d s}(y(s, t, \epsilon)-t)\right| \leqq K|\gamma(t)| e^{-\mu s / 2 \epsilon}+\omega(\epsilon)+K|y(s, t, \epsilon)-t| \\
\quad \text { if }(x, y) \varepsilon Q, \quad t_{0} \leqq t \leqq t_{1}, \quad|u| \leqq \tilde{\delta}_{1}, \quad 0<\epsilon \leqq \tilde{\epsilon}_{0} .
\end{gathered}
$$

Applying Lemma 3.2 to (4.20) yields

$$
\begin{aligned}
& |y(s, t, \epsilon)-t| \leqq \omega(\epsilon) \\
& \text { if }(x, y) \varepsilon Q, \quad t_{0} \leqq t \leqq t_{1}, \quad|u| \leqq \tilde{\delta}_{1}, \quad 0<\epsilon \leqq \tilde{\epsilon}_{0} .
\end{aligned}
$$

From (4.18) and (4.21) we now have

$$
\begin{aligned}
& |y(s, t, \epsilon)-t| \leqq \omega(\epsilon) \\
& |u(s, t, \epsilon)| \leqq 2|\gamma(t)| e^{-\mu s / 2 \epsilon}+\omega(\epsilon) \\
& \text { if }(x, y) \varepsilon Q, \quad t_{0} \leqq t \leqq t_{1}, \quad|u| \leqq \tilde{\delta}_{1}, \quad 0<\epsilon \leqq \tilde{\epsilon}_{0} .
\end{aligned}
$$

The conclusion of the lemma follows readily from (4.22).

Lemma 4.2. There exist constants $\delta_{2}$ and $\epsilon_{2}$, which are independent of $\epsilon$ and satisfy the inequalities

$$
0<\delta_{2} \leqq \delta_{1}, \quad 0<\epsilon_{2} \leqq \epsilon_{1}
$$


such that if

$$
|\gamma(t)| \leqq \delta_{2}, \quad t_{0} \leqq t \leqq t_{1},
$$

then

$$
\begin{aligned}
& \frac{\partial r}{\partial t}(s, t, \epsilon) \equiv 0 \\
& \left|\frac{\partial p}{\partial t}(s, t, \epsilon)-1\right| \leqq \omega(\epsilon) \\
& \left|\frac{\partial g}{\partial t}(s, t, \epsilon)\right| \leqq 2\left|\gamma^{\prime}(t)\right| e^{-\mu s / 2 \epsilon}+K|\gamma(t)| e^{-\mu s / 2 \epsilon}+\omega(\epsilon) \\
& \text { for } t_{0} \leqq t \leqq t_{1}, 0<\epsilon \leqq \epsilon_{2} .
\end{aligned}
$$

Proof. The existence of $\partial y(s, t, \epsilon) / \partial t$ and $\partial u(s, t, \epsilon) / \partial t$ is guaranteed by a well known theorem in differential equations. Differentiating (4.1) with respect to $t$ yields

$$
\begin{aligned}
& \frac{d}{d s}\left(\frac{\partial y}{\partial t}-1\right)=G_{3}\left(\frac{\partial y}{\partial t}-1\right)+G_{4} \frac{\partial u}{\partial t}+G_{3} \\
& \epsilon \frac{d}{d s}\left(\frac{\partial u}{\partial t}\right)=B(s, t) \frac{\partial u}{\partial t}+G_{5}\left(\frac{\partial y}{\partial t}-1\right)+G_{6} \frac{\partial u}{\partial t}+G_{5}
\end{aligned}
$$

where

$$
\begin{aligned}
& G_{3}(s, t, \epsilon)=a_{y}(s, y, u, \epsilon)-a_{y}(s, t, 0,0) \\
& G_{4}(s, t, \epsilon)=a_{u}(s, y, u, \epsilon) \\
& G_{5}(s, t, \epsilon)=b_{y}(s, y, u, \epsilon)-b_{y}(s, t, 0,0) \\
& G_{6}(s, t, \epsilon)=b_{u}(s, y, u, \epsilon)-b_{u}(s, t, 0,0) .
\end{aligned}
$$

In (4.25) we have used

$$
a_{y}(s, t, 0,0)=b_{y}(s, t, 0,0) \equiv 0,
$$

which follows immediately from $\mathrm{H}^{\prime}$ and $\mathrm{H}^{\prime}$.

Let $\nu>0$. From $H 1^{\prime}, \mathrm{H} 2^{\prime}$, and (4.4) there exist constants $\tilde{\delta}_{2}=\tilde{\delta}_{2}(\nu)$ and $\tilde{\epsilon}_{2}=\tilde{\epsilon}_{2}(\nu)$, satisfying $0<\tilde{\delta}_{2} \leqq \delta_{1}$ and $0<\tilde{\epsilon}_{2} \leqq \epsilon_{1}$, such that

$$
\begin{aligned}
& \left|G_{3}(s, t, \epsilon)\right| \leqq K|\gamma(t)| e^{-\mu s / 2 \epsilon}+\omega(\epsilon) \\
& \left|G_{4}(s, t, \epsilon)\right| \leqq K \\
& \left|G_{5}(s, t, \epsilon)\right| \leqq K|\gamma(t)| e^{-\mu s / 2 \epsilon}+\omega(\epsilon) \\
& \left|G_{6}(s, t, \epsilon)\right| \leqq \nu \\
& \text { if } t_{0} \leqq t \leqq t_{1}, \quad|\gamma(t)| \leqq \tilde{\delta}_{2}, \quad 0<\epsilon \leqq \tilde{\epsilon}_{2} .
\end{aligned}
$$


Exactly the same arguments used in the proof of Lemma 4.1 may now be applied to the system (4.24) and the related inequalities (4.26) in order to obtain (4.23). The constant $\nu$ may be taken equal to $\nu_{0}$. We omit the details.

Lemma 4.3. There exist constants $\delta_{3}$ and $\epsilon_{3}$, which are independent of $\epsilon$ and satisfy the inequalities

$$
0<\delta_{3} \leqq \delta_{2}, \quad 0<\epsilon_{3} \leqq \epsilon_{2},
$$

such that if

$$
|\gamma(t)| \leqq \delta_{3}
$$$$
t_{0} \leqq t \leqq t_{1}
$$

then

$$
\begin{aligned}
& \frac{\partial r}{\partial s}(s, t, \epsilon) \equiv 1 \\
& \left|\frac{\partial p}{\partial s}(s, t, \epsilon)\right| \leqq K|\gamma(t)| e^{-\mu s / 2 \epsilon}+\omega(\epsilon) \\
& \left|\frac{\partial g}{\partial s}(s, t, \epsilon)\right| \leqq \frac{1}{\epsilon}\{K|\gamma(t)|+\omega(\epsilon)\} e^{-\mu s / 2 \epsilon}+\omega(\epsilon) \\
& \text { for } t_{0} \leqq t \leqq t_{1}, \quad 0<\epsilon \leqq \epsilon_{3} .
\end{aligned}
$$

Proof. The first line of (4.27) is, of course, obvious. From H2', the second equation of (4.1), and (4.4) we easily obtain

$$
\begin{aligned}
& \left|\frac{\partial y}{\partial s}(s, t, \epsilon)\right| \leqq K|\gamma(t)| e^{-\mu s / 2 \epsilon}+\omega(\epsilon) \\
& \text { for } t_{0} \leqq t \leqq t_{1}, \quad 0<\epsilon \leqq \epsilon_{1},
\end{aligned}
$$

which disposes of the second line of (4.27).

The third equation of (4.1) is now written as

$$
\epsilon \frac{d u}{d s}=B(s, t) u+G(s, t, \epsilon),
$$

where

$$
G(s, t, \epsilon)=b(s, y, u, \epsilon)-b_{u}(s, t, 0,0) u .
$$

Dividing (4.29) by $\epsilon$ and applying Lemma 3.3 to the resulting equation yields

$$
u(s, t, \epsilon)=\gamma(t) \exp \left(\frac{1}{\epsilon} \int_{0}^{s} B(\xi, t) d \xi\right)
$$

$$
+\frac{1}{\epsilon} \int_{0}^{s} \exp \left(\frac{1}{\epsilon} \int_{\xi}^{s} B(\eta, t) d \eta\right) G(\xi, t, \epsilon) d \xi .
$$


Equation (4.31) is, of course, the same as equation (4.9). Differentiating (4.31) with respect to $s$ yields

$$
\begin{aligned}
\frac{\partial u}{\partial s}(s, t, \epsilon)=\frac{1}{\epsilon} B(s, t) \gamma(t) \exp & \left(\frac{1}{\epsilon} \int_{0}^{s} B(\xi, t) d \xi\right)+\frac{1}{\epsilon} G(s, t, \epsilon) \\
& +\frac{1}{\epsilon^{2}} B(s, t) \int_{0}^{s} \exp \left(\frac{1}{\epsilon} \int_{\xi}^{s} B(\eta, t) d \eta\right) G(\xi, t, \epsilon) d \xi .
\end{aligned}
$$

Integrating the last term by parts and combining various terms results in

$$
\begin{gathered}
\frac{\partial u}{\partial s}(s, t, \epsilon)=\frac{1}{\epsilon} \frac{B(s, t)}{B(0, t)} b(0, t, \gamma(t), \epsilon) \exp \left(\frac{1}{\epsilon} \int_{0}^{s} B(\xi, t) d \xi\right) \\
+\frac{1}{\epsilon} B(s, t) \int_{0}^{s}\left\{\frac{B(\xi, t) \frac{\partial G}{\partial \xi}(\xi, t, \epsilon)-G(\xi, t, \epsilon) \frac{\partial B}{\partial \xi}(\xi, t)}{B^{2}(\xi, t)}\right\} \\
\quad \exp \left(\frac{1}{\epsilon} \int_{\xi}^{s} B(\eta, t) d \eta\right) d \xi .
\end{gathered}
$$

An appraisal of $\partial G(s, t, \epsilon) / \partial s$ is now made. From (4.30) one has

$$
\begin{aligned}
\frac{\partial G}{\partial s}(s, t, \epsilon)=\left\{b_{x}(s, y, u, \epsilon)-b_{x}(s, t, 0,0)\right\} & +b_{y}(s, y, u, \epsilon) \frac{\partial y}{\partial s}+b_{u x}(s, t, 0,0) u \\
& +\left\{b_{u}(s, y, u, \epsilon)-b_{u}(s, t, 0,0)\right\} \frac{\partial u}{\partial s}
\end{aligned}
$$

where we have used

$$
b_{x}(s, t, 0,0) \equiv 0
$$

which follows from $\mathrm{H}^{\prime}$. Let $\nu>0$. From $\mathrm{H} 1^{\prime}$ and (4.4) there exist constants $\tilde{\delta}_{3}=\tilde{\delta}_{3}(\nu)$ and $\tilde{\epsilon}_{3}=\tilde{\epsilon}_{3}(\nu)$, satisfying $0<\tilde{\delta}_{3} \leqq \delta_{2}$ and $0<\tilde{\epsilon}_{3} \leqq \epsilon_{2}$, such that

$$
\begin{gathered}
\left|\frac{\partial G}{\partial s}(s, t, \epsilon)\right| \leqq K|\gamma(t)| e^{-\mu s / 2 \epsilon}+\omega(\epsilon)+\nu\left|\frac{\partial u}{\partial s}(s, t, \epsilon)\right| \\
\text { if } t_{0} \leqq t \leqq t_{1}, \quad|\gamma(t)| \leqq \tilde{\delta}_{3}, \quad 0<\epsilon \leqq \tilde{\epsilon}_{3} .
\end{gathered}
$$

Also, from $\mathrm{H1}^{\prime}$, (4.4), and (4.30) one has

$$
\begin{aligned}
& |G(s, t, \epsilon)| \leqq K|\gamma(t)| e^{-\mu s / 2 \epsilon}+\omega(\epsilon) \\
& \text { if } t_{0} \leqq t \leqq t_{1}, \quad|\gamma(t)| \leqq \delta_{1}, \quad 0<\epsilon \leqq \epsilon_{1} .
\end{aligned}
$$


Combining $\mathrm{H}^{\prime}$, (4.32), (4.33), and (4.34) we obtain

$$
\begin{gathered}
\left|\frac{\partial u}{\partial s}(s, t, \epsilon)\right| \leqq \frac{1}{\epsilon}\{K|\gamma(t)|+\omega(\epsilon)\} e^{-\mu s / \epsilon}+K|\gamma(t)| e^{-\mu s / 2 \epsilon}+\omega(\epsilon) \\
+\frac{K \nu}{\epsilon} \int_{0}^{s} e^{-\mu(s-\xi) / \epsilon}\left|\frac{\partial u}{\partial \xi}(\xi, t, \epsilon)\right| d \xi \\
\text { if } t_{0} \leqq t \leqq t_{1}, \quad|\gamma(t)| \leqq \tilde{\delta}_{3}, \quad 0<\epsilon \leqq \tilde{\epsilon}_{3} .
\end{gathered}
$$

Lemma 3.1 may now be applied to (4.35) in the usual way to obtain the last line of (4.27). We take $\nu=\nu_{1}$ so that $\mu-K \nu_{1}>\frac{1}{2} \mu$, where $K$ is the constant $K$ appearing in (4.35). We omit the details.

5. Proof of Theorem 2. Consider the functions

$$
x=r(s, t, \epsilon) \equiv s, \quad y=p(s, t, \epsilon)
$$

obtained in Section 4. If we can show that (5.1) can be solved for $s$ and $t$ in terms of $x, y$, and $\epsilon$ (for sufficiently small $\epsilon$ ), and then if we substitute these functions into

$$
u=g(s, t, \epsilon)
$$

obtained in Section 4, it follows from a well known theorem (see [1] pages 51 to 54 ) that we have obtained (for sufficiently small $\epsilon$ ) the unique solution of (2.10) passing through $\Gamma,(2.11)$. However, the solvability of (5.1) for $s$ and $t$ and the fact that the domain of definition of the solution of (2.10) passing through (2.11) contains $D$ (see the definition of $D$ following Theorem 2 ) for sufficiently small $\epsilon$ are evident from (4.4) and (4.23). Denote the solution of (5.1) by

Then

$$
s=x, \quad t=t(x, y, \epsilon)
$$

$$
H(x, y, \epsilon)=g(x, t(x, y, \epsilon), \epsilon)
$$

is the required solution of (2.10).

All that remains to be shown is the behavior of $H$ and its first partial derivatives as $\epsilon \rightarrow 0+$. Elementary calculations yield

$$
\begin{aligned}
& \frac{\partial H}{\partial x}(x, y, \epsilon)=\frac{\partial g}{\partial s}(s, t, \epsilon)-\frac{\frac{\partial p}{\partial s}(s, t, \epsilon)}{\frac{\partial p}{\partial t}(s, t, \epsilon)} \frac{\partial g}{\partial t}(s, t, \epsilon) \\
& \frac{\partial H}{\partial y}(x, y, \epsilon)=\frac{1}{\frac{\partial p}{\partial t}(s, t, \epsilon)} \frac{\partial g}{\partial t}(s, t, \epsilon) .
\end{aligned}
$$

The result now follows from (4.4), (4.23), (4.27), (5.3), and (5.4). 
6. The boundary layer equation. We have seen that the solution, $H(x, y, \epsilon)$, of (2.10) changes rapidly with $\epsilon$ in the neighborhood of $\Gamma,(2.11)$, while away from $\Gamma$ it is close to the surface $u=0$. This suggests the possibility of finding an equation which is "simpler" than (2.10) and whose solution is close to $H(x, y, \epsilon)$ in the neighborhood of $\Gamma$. With a knowledge only of the solution of this equation and the surface $u=0$, one would then have fairly complete information on $H(x, y, \epsilon)$. The equation

$$
\epsilon\left\{\frac{\partial \bar{u}}{\partial x}+a(0, t, \bar{u}, 0) \frac{\partial \bar{u}}{\partial y}\right\}=b(0, t, \bar{u}, 0),
$$

which we refer to as the boundary layer equation associated with (2.10) and $\Gamma$, immediately suggests itself as the simpler equation. It will be seen from the following that by using (6.1) we also obtain a somewhat more natural approach to the maximum permissible value of $|\gamma(t)|$ than the $\hat{\delta}$ of Theorem 2. The improved version of Theorem 2 thereby obtained is stated as Theorem 3 and appears at the end of this section. Much of the proof of Theorem 3 is the same as that of Theorem 2 and therefore will not be repeated here.

Hypotheses $\mathrm{H}^{\prime}, \mathrm{H}^{\prime}$, and $\mathrm{H}^{\prime}{ }^{\prime}(\mathrm{i})$ are again assumed (H3'(ii) is dropped) besides an additional one, $\mathrm{H}^{\prime}$, that will be given shortly.

The characteristic equations associated with (6.1) are

$$
\begin{aligned}
\frac{d x}{d s} & =1 \\
\frac{d y}{d s} & =a(0, t, \bar{u}, 0) \\
\epsilon \frac{d \bar{u}}{d s} & =b(0, t, \bar{u}, 0),
\end{aligned}
$$

and their solutions are denoted by

$$
\begin{aligned}
& x=\bar{r}(s, t, \epsilon) \equiv s \\
& y=\bar{p}(s, t, \epsilon) \\
& u=\bar{g}(s, t, \epsilon) .
\end{aligned}
$$

The associated initial conditions are given by

$$
\begin{array}{ll}
\bar{r}(0, t, \epsilon)=0 & \\
\bar{p}(0, t, \epsilon)=t, & t_{0} \leqq t \leqq t_{\mathbf{1}} . \\
\bar{g}(0, t, \epsilon)=\gamma(t) &
\end{array}
$$

The change of variable

$$
\rho=s / \epsilon
$$


shows that $\bar{g}$ satisfies the equation

$$
\frac{d \bar{g}}{d \rho}=b(0, t, \bar{g}, 0) \text {. }
$$

It is convenient to use the notation $\bar{p}(\epsilon \rho, t, \epsilon)=\bar{p}(\rho, t, \epsilon), \bar{g}(\epsilon \rho, t, \epsilon)=\bar{g}(\rho, t)$, which should cause no confusion, as it will be clear from the context whether we are using the variables $\rho, t, \epsilon$ or $s, t, \epsilon$.

$\mathrm{H} 4^{\prime}$ : The unique solution, $\bar{g}=\bar{g}(\rho, t)$, of (6.6) satisfying the initial condition $\bar{g}(0, t)=\gamma(t)$ exists for all $\rho \geqq 0, t_{0} \leqq t \leqq t_{1}$; and $|\bar{g}(\rho, t)| \rightarrow 0$ as $\rho \rightarrow+\infty$ uniformly with respect to $t$ for $t_{0} \leqq t \leqq t_{1}$. Furthermore, there exists a region, $R^{\prime}$, of the $x, y, u, \epsilon$-space which contains in its interior the set $x=0, y=t$, $u=\bar{g}(\rho, t), \epsilon=0$, where $0 \leqq \rho<\infty$ and $t_{0} \leqq t \leqq t_{1}$, such that in $R^{\prime}: a$ and $b$ and their second partial derivatives with respect to $x, y$, and $u$ are continuous in $(x, y, u, \epsilon)$.

From $\mathrm{H}^{\prime}$ it can easily be shown that once $|\bar{g}(\rho, t)|$ gets sufficiently small then $|\bar{g}(\rho, t)| \rightarrow 0$ as $\rho \rightarrow+\infty$ uniformly with respect to $t$ for $t_{0} \leqq t \leqq t_{1}$. Thus part of $\mathrm{H} 4^{\prime}$ is implied by $\mathrm{H} 1^{\prime}$.

Well known theorems on differentiability with respect to initial conditions and parameters insures that $\partial \bar{g}(\rho, t) / \partial t$ is continuous in $(\rho, t)$ for $0 \leqq \rho<\infty$, $t_{0} \leqq t \leqq t_{1}$.

It is emphasized that from now on $\gamma(t)$ is the fixed function of (6.4) and $\mathrm{H}^{\prime}$.

Lemma 6.1. Let $\rho_{1}>0$ be given. Then there exists an $\epsilon_{1}\left(\rho_{1}\right)>0$ such that

$$
x=s, \quad y=\bar{p}(s, t, \epsilon)
$$

may be solved for $s$ and $t$, when $0 \leqq s \leqq \epsilon \rho_{1}, t_{0} \leqq t \leqq t_{1}, 0<\epsilon \leqq \epsilon_{1}\left(\rho_{1}\right)$.

Proof. From the second equations of (6.2) and (6.4) we have

$$
\bar{p}(\rho, t, \epsilon)=t+\epsilon \int_{0}^{\rho} a(0, t, \bar{g}(\xi, t), 0) d \xi .
$$

Then

$$
\frac{\partial \bar{p}}{\partial t}(\rho, t, \epsilon)=1+\epsilon \int_{0}^{\rho} \frac{\partial}{\partial t} a(0, t, \bar{g}(\xi, t), 0) d \xi
$$

Hence, there exists an $\epsilon_{1}\left(\rho_{1}\right)>0$ such that

$$
\begin{gathered}
\frac{\partial \bar{p}}{\partial t}(s, t, \epsilon) \neq 0 \\
\text { for } 0 \leqq s \leqq \epsilon \rho_{1}, \quad t_{0} \leqq t \leqq t_{1}, \quad 0<\epsilon \leqq \epsilon_{1}\left(\rho_{1}\right) .
\end{gathered}
$$

The conclusion follows immediately from $x \equiv s$ and (6.10).

Define $\bar{D}\left(\epsilon, \rho_{1}\right)$ as the image of the set

$$
0 \leqq s \leqq \epsilon \rho_{1}, \quad t_{0} \leqq t \leqq t_{1}
$$

in the $x, y$-plane under the mapping (6.7). 
Corollary 6.1. Equation (6.1) has a unique solution passing through $\Gamma$ for $0<\epsilon \leqq \epsilon_{1}\left(\rho_{1}\right)$. The region of definition of this solution contains $\bar{D}\left(\epsilon, \rho_{1}\right)$.

Proof. Substituting the functions obtained in Lemma 6.1 into

$$
\bar{u}=\bar{g}(s, t, \epsilon),
$$

we obtain the solution of (6.1) passing through $\Gamma$. The $t$ in (6.1) is an added complication and requires us to use a slight generalization of the theorem previously referred to in [1]. The proof of the generalized theorem requires no new arguments.

It should be observed that the domain of definition of the solution of (6.1) just obtained may become arbitrarily small as $\epsilon \rightarrow 0+$. However, and this is the important thing, by taking $\rho_{1}$ sufficiently large the solution of (6.1) comes as close to the plane $u=0$ as we choose.

The functions $r(s, t, \epsilon) \equiv s, p(s, t, \epsilon)$, and $g(s, t, \epsilon)$ are again defined as the solution of (4.1) satisfying the initial condition (4.3), where $\gamma(t)$ is now the function of (6.4) and $\mathrm{H}^{\prime}$, however. In the following lemma the existence, uniqueness, and relationship to $\bar{r}, \bar{p}$, and $\bar{g}$ of $r, p$, and $g$ is established.

In the following $K\left(\rho_{1}\right)$ shall denote a positive number that depends only on $\rho_{1}$; this convention is similar to that used in connection with $K$.

Lemma 6.2. There exists an $\epsilon_{2}\left(\rho_{1}\right)$, which satisfies the inequality

$$
0<\epsilon_{2}\left(\rho_{1}\right) \leqq \epsilon_{1}\left(\rho_{1}\right),
$$

such that $r(s, t, \epsilon), p(s, t, \epsilon), g(s, t, \epsilon)$ exist, are unique, and satisfy

$$
\begin{aligned}
& r(s, t, \epsilon)=\bar{r}(s, t, \epsilon) \equiv s \\
& |p(s, t, \epsilon)-\bar{p}(s, t, \epsilon)| \leqq K\left(\rho_{1}\right) \epsilon \omega(\epsilon) \\
& |g(s, t, \epsilon)-\bar{g}(s, t, \epsilon)| \leqq K\left(\rho_{1}\right) \omega(\epsilon) \\
& \left|\frac{\partial p}{\partial t}(s, t, \epsilon)-\frac{\partial \bar{p}}{\partial t}(s, t, \epsilon)\right| \leqq K\left(\rho_{1}\right) \epsilon \omega(\epsilon) \\
& \left|\frac{\partial g}{\partial t}(s, t, \epsilon)-\frac{\partial \bar{g}}{\partial t}(s, t, \epsilon)\right| \leqq K\left(\rho_{1}\right) \omega(\epsilon) \\
& \left|\frac{\partial p}{\partial s}(s, t, \epsilon)-\frac{\partial \bar{p}}{\partial s}(s, t, \epsilon)\right| \leqq K\left(\rho_{1}\right) \omega(\epsilon) \\
& \left|\frac{\partial g}{\partial s}(s, t, \epsilon)-\frac{\partial \bar{g}}{\partial s}(s, t, \epsilon)\right| \leqq K\left(\rho_{1}\right) \omega(\epsilon) / \epsilon \\
& \text { for } 0 \leqq s \leqq \epsilon \rho_{1}, \quad t_{0} \leqq t \leqq t_{1}, \quad 0<\epsilon \leqq \epsilon_{2}\left(\rho_{1}\right) .
\end{aligned}
$$

Proof. The local existence and uniqueness of $r, p, g$ are guaranteed by well 
known theorems. The first line of $(6.11)$ is obvious. Define $\alpha(s, t, \epsilon)$ and $\beta(s, t, \epsilon)$ by

$$
\begin{aligned}
& \alpha=p(s, t, \epsilon)-\bar{p}(s, t, \epsilon) \\
& \beta=g(s, t, \epsilon)-\bar{g}(s, t, \epsilon) .
\end{aligned}
$$

It is clear that $\alpha$ and $\beta$ satisfy the equations

$$
\frac{d \alpha}{d \rho}=\epsilon\{a(\epsilon \rho, \bar{p}+\alpha, \bar{g}+\beta, \epsilon)-a(0, t, \bar{g}, 0)\}
$$

$$
\frac{d \beta}{d \rho}=b(\epsilon \rho, \bar{p}+\alpha, \bar{g}+\beta, \epsilon)-b(0, t, \bar{g}, 0)
$$

and the initial conditions

$$
\begin{aligned}
& \alpha(0, t, \epsilon)=0 \\
& \beta(0, t, \epsilon)=0 .
\end{aligned}
$$

From $H 4^{\prime}$ and (6.8) there exist constants $\hat{\delta}_{0}>0$ and $\hat{\epsilon}_{0}>0$ such that

$$
\begin{aligned}
& \left|\frac{d \alpha}{d \rho}\right| \leqq K\left(\rho_{1}\right) \epsilon\{|\alpha|+|\beta|+\omega(\epsilon)\} \\
& \left|\frac{d \beta}{d \rho}\right| \leqq K\left(\rho_{1}\right)\{|\alpha|+|\beta|+\omega(\epsilon)\}
\end{aligned}
$$

if $0 \leqq \rho \leqq \rho_{1}, \quad t_{0} \leqq t \leqq t_{1}, \quad|\alpha| \leqq \hat{\delta}_{0}, \quad|\beta| \leqq \hat{\delta}_{0}, \quad 0<\epsilon \leqq \hat{\epsilon}_{0}$.

We assume, without loss of generality, that $\epsilon_{1}\left(\rho_{1}\right) \leqq \hat{\epsilon}_{0}$. From (6.14) and (6.15) one has

$$
\left|\frac{d \beta}{d \rho}\right| \leqq K\left(\rho_{1}\right)\{|\beta|+\omega(\epsilon)\}
$$

$$
\text { if } 0 \leqq \rho \leqq \rho_{1}, \quad t_{0} \leqq t \leqq t_{1}, \quad|\alpha| \leqq \hat{\delta}_{0}, \quad|\beta| \leqq \hat{\delta}_{0}, \quad 0<\epsilon \leqq \hat{\epsilon}_{0} .
$$

Applying Lemma 3.2 to (6.16) one obtains

$$
\begin{aligned}
& |\beta(\rho, t, \epsilon)| \leqq K\left(\rho_{1}\right) \omega(\epsilon) \\
& \text { if } 0 \leqq \rho \leqq \rho_{1}, \quad t_{0} \leqq t \leqq t_{1}, \quad|\alpha| \leqq \hat{\delta}_{0}, \quad|\beta| \leqq \hat{\delta}_{0}, \quad 0<\epsilon \leqq \hat{\epsilon}_{0} .
\end{aligned}
$$

From (6.15) and (6.17) we have

$$
\left|\frac{d \alpha}{d \rho}\right| \leqq K\left(\rho_{1}\right) \epsilon\{|\alpha|+\omega(\epsilon)\}
$$

if $0 \leqq \rho \leqq \rho_{1}, \quad t_{0} \leqq t \leqq t_{1}, \quad|\alpha| \leqq \hat{\delta}_{0}, \quad|\beta| \leqq \hat{\delta}_{0}, \quad 0<\epsilon \leqq \hat{\epsilon}_{0}$. 
Applying Lemma 3.2 to (6.18) yields

$$
\begin{aligned}
& |\alpha(\rho, t, \epsilon)| \leqq K\left(\rho_{1}\right) \epsilon \omega(\epsilon) \\
& \text { if } 0 \leqq \rho \leqq \rho_{1}, \quad t_{0} \leqq t \leqq t_{1}, \quad|\alpha| \leqq \hat{\delta}_{0}, \quad|\beta| \leqq \hat{\delta}_{0}, \quad 0<\epsilon \leqq \hat{\epsilon}_{0} .
\end{aligned}
$$

Inequalities (6.17) and (6.19) are easily seen to imply the second and third lines of (6.11). Substituting (6.17) and (6.19) into (6.15) then yields the last two inequalities of (6.11).

Differentiating (6.13) with respect to $t$ and applying $H 4^{\prime},(6.9),(6.17)$, and (6.19) to the resulting equations yields after a little computation

$$
\begin{aligned}
& \left|\frac{d}{d \rho}\left(\frac{\partial \alpha}{\partial t}\right)\right| \leqq K\left(\rho_{1}\right) \epsilon\left\{\left|\frac{\partial \alpha}{\partial t}\right|+\left|\frac{\partial \beta}{\partial t}\right|+\omega(\epsilon)\right\} \\
& \left|\frac{d}{d \rho}\left(\frac{\partial \beta}{\partial t}\right)\right| \leqq K\left(\rho_{1}\right)\left\{\left|\frac{\partial \alpha}{\partial t}\right|+\left|\frac{\partial \beta}{\partial t}\right|+\omega(\epsilon)\right\} \\
& \text { for } 0 \leqq \rho \leqq \rho_{1}, \quad t_{0} \leqq t \leqq t_{1}, \quad 0<\epsilon \leqq \epsilon_{2}\left(\rho_{1}\right) .
\end{aligned}
$$

Essentially the same reasoning that led from (6.15) to (6.17) and (6.19) now yields

$$
\begin{aligned}
& \left|\frac{\partial \alpha}{\partial t}(s, t, \epsilon)\right| \leqq K\left(\rho_{1}\right) \epsilon \omega(\epsilon) \\
& \left|\frac{\partial \beta}{\partial t}(s, t, \epsilon)\right| \leqq K\left(\rho_{1}\right) \omega(\epsilon) \\
& \text { for } 0 \leqq s \leqq \epsilon \rho_{1}, \quad t_{0} \leqq t \leqq t_{1}, \quad 0<\epsilon \leqq \epsilon_{2}\left(\rho_{1}\right)
\end{aligned}
$$

when applied to (6.20). Inequalities (6.21) are the fourth and fifth lines of (6.11).

Lemma 6.3. There exists an $\epsilon_{3}\left(\rho_{1}\right)$, which satisfies the inequality

such that

$$
0<\epsilon_{3}\left(\rho_{1}\right) \leqq \epsilon_{2}\left(\rho_{1}\right)
$$

$$
x=s, \quad y=p(s, t, \epsilon)
$$

may be solved for $s$ and $t$, when $0 \leqq s \leqq \epsilon \rho_{1}, t_{0} \leqq t \leqq t_{1}, 0<\epsilon \leqq \epsilon_{3}\left(\rho_{1}\right)$.

Proof. From (6.9) and (6.21) one easily obtains an $\epsilon_{3}\left(\rho_{1}\right)$, satisfying

$$
0<\epsilon_{3}\left(\rho_{1}\right) \leqq \epsilon_{2}\left(\rho_{1}\right),
$$

such that

$$
\frac{\partial p}{\partial t}(s, t, \epsilon) \neq 0
$$

for $0 \leqq s \leqq \epsilon \rho_{1}, \quad t_{0} \leqq t \leqq t_{1}, \quad 0<\epsilon \leqq \epsilon_{3}\left(\rho_{1}\right)$.

The conclusion follows immediately from $x \equiv s$ and (6.23). 
Define $D\left(\epsilon, \rho_{1}\right)$ as the image of the set

$$
0 \leqq s \leqq \epsilon \rho_{1}, \quad t_{0} \leqq t \leqq t_{1}
$$

in the $x, y$-plane under the mapping (6.22).

Corollary 6.3. Equation (2.10) has a unique solution passing through $\Gamma$ for $0<\epsilon \leqq \epsilon_{3}\left(\rho_{1}\right)$. The region of definition of this solution contains $D\left(\epsilon, \rho_{1}\right)$.

Proof. Substitute the functions obtained in Lemma 6.3 into

$$
u=g(s, t, \epsilon) .
$$

Theorem 3. $\mathrm{H}^{\prime}, \mathrm{H} 2^{\prime}, \mathrm{H} 3^{\prime}(\mathrm{i})$, and $\mathrm{H} 4^{\prime}$ imply that there exists a closed non-vacuous region, $D$ (which is independent of $\epsilon$ ) and a constant $\hat{\epsilon}>0$ (independent of $\epsilon$ ) such that (2.10) has a unique solution $u=H(x, y, \epsilon)$ which is defined over $D$ and satisfies the initial condition $H(0, t, \epsilon)=\gamma(t)$ for $t_{0} \leqq t \leqq t_{1}, 0<\epsilon \leqq \hat{\epsilon}$. Furthermore, $H(x, y, \epsilon) \rightarrow 0, \partial H(x, y, \epsilon) / \partial x \rightarrow 0$, and $\partial H(x, y, \epsilon) / \partial y \rightarrow 0$ as $\epsilon \rightarrow 0+$ uniformly for $x \geqq C$ for any $C>0$.

Proof. The $D$ of Theorem 3 is the same as the $D$ of Theorem 2. The proof is simply an application of Corollary 6.3 and the technique used in proving Theorem 2. If $\rho_{1}$ is taken sufficiently large and $\epsilon$ sufficiently small it follows from $\mathrm{H} 4^{\prime}$ and (6.11) that $\left|g\left(\epsilon \rho_{1}, t, \epsilon\right)\right|$ may be taken arbitrarily small. Hence,

$$
\Gamma(\epsilon):\left\{\begin{array}{l}
x=\epsilon \rho_{1} \\
y=p\left(\epsilon \rho_{1}, t, \epsilon\right), \quad t_{0} \leqq t \leqq t_{1} \\
u=g\left(\epsilon \rho_{1}, t, \epsilon\right)
\end{array}\right.
$$

may be used in the same role as $\Gamma,(2.11)$, i.e. it serves as an initial curve for (2.10). With respect to the system (4.1), we may now prove lemmas analogous to those of Section 4 in the subset of $Q$ to the right of $x=\epsilon \rho_{1}$. Only very minor and obvious changes are caused by the dependence of $\Gamma(\epsilon)$ on $\epsilon$. The proof is completed by the same arguments as those given in Section 5 .

\section{BIBLIOGRAPHY}

[1] R. Courant \& D. Hilbert, Methoden der mathematischen Physik, Vol. 2, Berlin (1937). [2] J. J. Levin \& N. Levinson, Singular perturbations of non-linear systems of differential equations and an associated boundary layer equation, This Journal, 3, 247-270 (1954). 